\title{
The Study on the Influencing Factors of Green Consumption Behavior in Shanghai High-Star Hotels
}

\author{
Jiang Bing-rui ${ }^{1, a^{*}}$, Zhou Jia-yu ${ }^{2, b}$ \\ ${ }^{1}$ Department of Tourism Management, Shanghai Sanda University, Pudong District, Shanghai, China \\ ${ }^{2}$ Department of Hospitality Management, Shanghai Sanda University, Pudong District, Shanghai, China \\ a*15755351927@126.com \\ b1264813680@qq.com
}

\begin{abstract}
This research aims to explore the influencing factors of hotel customers' green consumption behavior, and analyze the mechanism of influencing factors on customers' green consumption behavior. Based on the results of a questionnaire survey of 413 customers in high-star hotels in Shanghai and uses exploratory factor analysis and multiple regression analysis to explore the influencing factors and mechanism of hotel customers' green consumption behavior. The results show that: Green consumption behavior includes general behavior and positive behavior. Subjective norms and perceived profits have significant positive effects and high explanatory power on general and positive green consumption behavior. Perceived profit and perceived loss have significant negative impact on general behavior and some positive behaviors, which together with perceived profit reflect the internal psychological attribution of consumers' green consumption behavior; The green consumption situation has higher explanatory effect on the green consumption behavior related to the lodging experience. Compared with previous studies, high-star hotel customers are more willing to take general behavior and positive behavior than general hotel public, and subjective norms have higher explanatory effect on general behavior and positive behavior.
\end{abstract}

Keywords: Green consumption behavior, influencing factors, high-star hotel, exploratory factor analysis, multiple regression analysis

\section{上海市高星级酒店顾客绿色消费行为影响因素研究 江丙瑞 ${ }^{1, a}$ 周嘉矩 ${ }^{2, b *}$}

\author{
上海杉达学院旅游管理系, 浦东新区, 上海, 中国

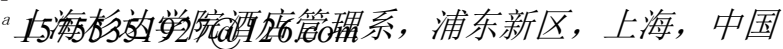 \\ b*1264813680@qq.com
}

\section{摘要}

本文旨在探究酒店顾客绿色消费行为的影响因素, 分析影响因素对顾客绿色消费行为的作用机制, 并基于上海 高星级酒店 413 位顾客的问卷调查数据，运用探索性因子分析法与多元回归分析法探究酒店顾客绿色消费行 为影响因素及作用机制。研究结果显示: 主观规范与感知利得对一般及积极绿色消费行为均存在显著正向影 响, 且具有较高的解释力; 感知利失对一般行为与部分积极行为表现出显著负向影响, 与感知利得共同体现了 消费者绿色消费行为的内部心理归因; 绿色消费情境对与入住体验有关的绿色消费行为具有较高的解释效力。 与以往研究相比, 高星级酒店顾客较一般酒店公众在一般行为与积极行为意愿更为强烈, 且主观规范对其采取 一般行为及积极行为均有较高解释效力。

关键词: 绿色消费行为; 影响因素; 高星级酒店; 探索性因子分析; 多元回归分析 


\section{1. 前言}

据世界旅游组织相关报告显示: 全球 5\%因旅游业 排放的二氧化碳中， $2 \%$ 源自于酒店宾馆等住宿业 ${ }^{[1]}$ 。 住宿业的能源使用密集性可见一斑, 正如此, 建设与 经营 “绿色酒店” 成为越来越多酒店企业主的追求 的目标。但仅依托环保设备、技术以及酒店企业主的 绿色经营理念实现行业绿色发展远远不够, 顾客作为 酒店服务与产品的最终消费者, 是否配合与支持酒店 绿色消费措施的推行具有关键影响。因此, 在绿色发 展方式与生活方式成为时代发展主流趋势下, 准确把 握顾客配合与支持绿色消费行为的影响因素及行为 机理对行业绿色发展新面貌的塑造具有一定的参考 价值。

\section{2. 文献回顾与问题提出}

绿色消费行为, 又称环境友好行为, 它是指社会 个体通过价值选择和自我认同所构建的, 兼顾消费目 标达成与减少环境破坏的消费方式，包括产品的购买、 使用和处置三个环节 ${ }^{[2]}$ 。在酒店消费情境下, 以顾客 入住绿色酒店决策机制的实证研究居多 ${ }^{[3-4]}$ 。一方面, 基于计划行为理论、价值一信仰-规范等理论对酒店顾 客绿色消费行为进行实证分析 ${ }^{[5-6]}$; 另一方面, 独立于 传统理论模型之外的绿色酒店消费意愿的影响因素 研究受到西方学者的较多关注, 如环境关注 ${ }^{[7]}$ 、日常 环保行为 ${ }^{[8]}$ 、绿色信任 ${ }^{[4]}$ 等。显然, 国内外学术界关于 酒店消费情境下的绿色消费行为研究相当成熟, 但鲜 有研究关注到高星级酒店这一特定领域。高星级酒店 顾客注重消费体验, 对酒店软硬件设施与服务要求较 高, 在这一细分消费情境下: 个体采取绿色消费行为 的影响因素有哪些? 各因素对相关绿色消费行为的 作用机制如何? 进一步地, 高星级酒店顾客与以往研 究中的酒店顾客绿色消费行为存在哪些差异? 基于 此, 本文通过探究酒店顾客绿色消费行为的影响因素, 分析影响因素对顾客绿色消费行为的作用机制, 在对 比前人研究基础上, 尝试回答上述问题。

\section{3. 研究设计}

\section{1 问卷设计}

本文采用问卷调查的方式收集数据。问卷设计上, 主要吸纳赵黎明等 ${ }^{[9]}$ 、李祝平 ${ }^{[10]} 、$ Choi 等 ${ }^{[4]}$ 、Nimri 等 ${ }^{[11]} 、$ Mas' $\mathrm{od}^{[8]}$ 、Kang 等 ${ }^{[7]}$ 前人研究的量表结构和题项, 一定程度上能保证量表的内容效度 ${ }^{[12]}$ 。在此基础上, 要求相关专家对题项重要性进行打分, 最终提炼出 30 个题项。其中, 基本人口学特征题项 4 个, 量表题项 26 个, 前置变量 22 个题项, 由态度、绿色消费知识、 主观规范、感知利益、绿色消费情境、日常环保行为 等维度构成。结果变量方面, 绿色消费行为由一般行 为与积极行为构成, 一般行为题项包括 “我会配合酒 店绿色消费措施 (如减少易耗品使用和床单更换频率 等) (Y1)”、“我未来会向亲朋好友宣传介绍酒店的 相关绿色环保措施（Y2）”，积极行为包括 “我会选
择经过认证的绿色酒店入住（Y3）”、“我愿意付费 支持绿色消费措施 (Y4)”。所有题项均采用 “1-5(完 全赞同-完全不赞同）”五点李克特量表形式在问卷 中展示。

\section{2 数据来源与收集}

问卷面向上海高星级酒店住客进行发放，发放酒 店有上海豫园万丽酒店、上海五角场凯悦酒店、上海 金茂君悦大酒店和上海浦西四季酒店等四家高星级 酒店，截止 2020 年 2 月 17 日，历时 2 个月，共收到 457 份问卷, 剔除填写时间低于 2 分钟的问卷，共得 到有效问卷 413 分, 有效率 92. 9\%。其中, 男性占比 $50.6 \%$ ，女性 49. 4\% ; 年龄上，19-45 岁年龄段顾客占 $87.2 \%$, 住客年龄整体偏年轻化。受教育程度上, $75.7 \%$ 顾客受教育程度在本科及以上, 住客整体受教育水平 较高。来源地上, 以长三角省份顾客居多。

\section{3 分析方法}

本文利用探索性因子分析法确定影响高星级酒 店顾客绿色消费行为的主要驱动因素, 并进一步利用 多元线性回归分析法探求驱动因素与绿色消费行为 的统计关系。

\section{4. 结果与分析}

\section{1 消费行为影响因素探析}

在确保适合进行因子分析的前提下, 本文采用主 成分分析方法对数据进行公因子萃取, 最终保留了 14 个题项, 且汇聚为 4 个因子结构, 累计方差贡献率为 $70.585 \%$ ，概括了 14 个题项 7 成以上信息。因子 1 包 含 5 个题项, 题项反映了亲朋好友、他人等参考群体 对个人行为的影响, 沿用原预设维度 “主观规范” ; 因子 2 包括 5 个题项，反映出酒店顾客期望在绿色消 费行为中得到益处, 故命名为 “感知利得” ; 因子 3 包括 2 个题项, 反映酒店顾客绿色消费行为需要特定 情境的 “驯化” ，沿用原预设维度 “绿色消费情境” ; 因子 4 包括 2 个题项，体现了顾客认为绿色消费可能 带来的负面影响, 故命名为 “感知利失”。此外, 比 较积极行为与一般行为均值发现, 一般行为 (Y1、Y2 分别为 $2.03,2.11$ ) 与积极行为的意愿水平 ( $\mathrm{Y} 3, \mathrm{Y} 4$ 分别为 $2.06,2.16$ ) 较一致, 一般行为意愿略高于积 极行为。

\section{2 消费行为影响因素的回归分析}

为揭示各影响因子对高星级酒店顾客绿色消费 行为的作用机制，本文拟利用多元回归分析构建回归 模型。共线性诊断结果显示: 容忍度、方程膨胀系数 VIF 分别处于 0.255-0.457、2.189-3.916 范围，表 明进入回归方程式的自变量间多元共线性问题不是 很明显 ${ }^{[13]}$ 。且不同模拟方程中，显著性检验 $\mathrm{p}$ 值均为 0.000 , 均通过了 $5 \%$ 的显著性检验, 表明回归模型整 
体解释变异量达到显著水平。

\subsection{1 顾客一般行为影响因素的回归分析}

4 个影响因子可解释 “我会配合酒店绿色消费措 施 (Y1) ” 53.0\%变异量。非标准化多元回归方程为 “Y $1=0.284 *$ 主观规范 $+0.333 *$ 感知利得 $+0.191 *$ 绿色 消费情境 $-0.147 *$ 感知利失 +0.941 ”。其中，“主观规 范”、“感知利得”、“绿色消费情境”、“感知利 失” 对该行为均表现出显著影响, 其中, “主观规 范”、“感知利得” 和 “绿色消费情境” 正向影响因 变量, 而 “感知利失” 对因变量影响明显为负向。4 个显著回归系数的影响因子中, “主观规范”、“感 知利得” 的 $\beta$ 系数绝对值较大, 表明这两个影响因 子对 $Y 1$ 具有较高的解释力。

4 个影响因子可解释 “我未来会向亲朋好友宣传 介绍酒店的相关绿色环保措施 (Y2)” 55.0\%变异量。 非标准化多元回归方程为 “ $Y 2=0.471 *$ 主观规范 $+0.188 *$ 感知利得 $+0.050 *$ 绿色消费情境 $-0.234 *$ 感知 利失+1.542”, 其中, 仅 “主观规范” “ “感知利得” 和 “感知利失” 表现出显著影响, 其中, “主观规范” 和 “感知利得” 正向影响因变量, “感知利失” 负向 影响因变量。 $\beta$ 系数绝对值较大的影响因子 “主观规 范”与 “感知利失” 对 Y 2 具有较高的解释力。

\subsection{2 顾客积极行为影响因素的回归分析}

4 个自变量可解释 “我会选择经过认证的绿色酒 店入住 (Y3)” 54.9\%变异量。非标准化多元回归方程 为 “ $Y 3=0.262 *$ 主观规范 $+0.404 *$ 感知利得 $+0.186 *$ 绿 色消费情境 -0.126 *感知利失 +0.799 ”, 其中, “主观 规范” 、“感知利得” 和 “绿色消费情境” 呈显著正 向影响, 而 “感知利失” 则显著负向影响该行为。 $\beta$ 系数绝对值较大的 “感知利得”、“主观规范” 对 Y3 具有较高的解释力。

4 个自变量可解释 “我愿意付费支持绿色消费措 施 (Y4) ” 50.1\%变异量。非标准化多元回归方程为 “Y $4=0.672 *$ 主观规范 $+0.176 *$ 感知利得 $-0.003 *$ 绿色 消费情境 -0.066 *感知利失 +0.647 ”, 其中, 仅 “主观 规范” 和 “感知利得” 表现出显著正向影响。“绿色 消费情境”、“感知利失” 则表现出显著负向影响。 同时, $\beta$ 系数绝对值较大的 “主观规范” 对 Y4 具有 高解释力。

\subsection{3 影响因素及其作用机制讨论}

（1）而绿色消费知识和日常环保行为等预设变 量未得到汇聚。有研究证实, 绿色消费相关知识显著 影响消费者绿色消费行为 ${ }^{[10]}$, 亦有研究指出, 即便大 众传媒运动能提高消费者对于气候变暖的知识水平, 往往也不会带来显著的行为变迁 ${ }^{[14]}$ 。因此, 绿色消费 措施的推行与传播可能会提升大众对绿色消费的关 注, 但较难引起绿色消费知识水平的提升, 即使有提
升, 也无法对行为变迁产生明显影响。日常环保行为 虽有一定实践（题项均值在 2.02-2.13 范围), 然而 高星级酒店营造的奢华居住氛围异于惯常生活, 高质 量住宿体验成为消费者基本诉求。这一矛盾可能成为 此处无法观察到日常环保行为与高星级酒店顾客绿 色消费行为间关联的主要原因。

(2) 高星级酒店顾客一般行为与积极行为意愿 整体较强, 与以往研究结论相悖 ${ }^{[9]}$, 这或是高星级酒 店顾客在绿色消费知识、责任感等方面与一般酒店公 众存在明显差异导致, 且与近年来强调的绿色发展方 式和生活方式对社会公众的影响不无关系。

(3) 主观规范对一般行为与积极行为均存在显 著正向影响, 这与前人研究结论基本一致 ${ }^{[3-4]}$ 。人的行 为容易受到所处情境影响, 在知觉、认识和判断上易 趋从于周围人的行为方式, 产生趋同心理 ${ }^{[15]}$ 。且主观 规范对一般行为与积极行为均有较高解释效力, 这与 以往研究结论有所不同 ${ }^{[10]}$, 高星级酒店较一般酒店公 众而言, 亲朋等参考群体的行为、社会环境的压力对 个体在酒店消费情境下采取绿色消费行为有更为强 烈的引导作用。

（4）感知利得对绿色消费行为均存在显著正向 影响, 感知利失对部分绿色消费行为存在显著负向影 响。前者体现了酒店顾客采取相关行为时价值诉求与 声望寻求的心理归因, 它对 “我未来会向亲朋好友宣 传介绍酒店的相关绿色环保措施” 和 “我愿意付费 支持绿色消费措施” 解释效力不够突出, 这可能是由 行为与心理归因间契合度不高导致。感知利失体现了 人们关注绿色消费行为背后的 “收益” 问题。该因子 对相关行为呈负向影响, 如显著负向影响 “我未来会 向亲朋好友宣传介绍酒店的相关绿色环保措施” , 且 对该行为具有较大解释效力。这可能意味着, 顾客感 知 “收益” 越低, 越有可能向亲朋好友传达这一内容, 以帮助他们 “止损” 。总体来说, 无论感知利得或感 知利失, 一定程度上均是个体心理意识的体现, 属于 深层次的自我价值规范 ${ }^{[12]}$ 。

（5）绿色消费情境对 “我会配合酒店绿色消费 措施” 与 “我会选择经过认证的绿色酒店入住” 呈 显著正向影响。相关研究也有类似结论, 即单独使用 情境结构变革措施对现实社会中的消费者不具有说 服力, 传播沟通教育措施的配套使用也非常重要 ${ }^{[16]}$ 。 因此, 酒店情境营造、绿色消费产品的供给或许更有 利于刺激消费者产生行为迁移。

\subsection{4 未来研究方向讨论}

本文理论贡献在于: 以往研究多关注一般酒店公 众绿色消费行为, 缺少对高星级酒店这一特定领域绿 色消费行为影响因素及其作用机制研究; 其次, 从一 般行为和积极行为两方面分别探讨绿色消费行为, 通 过实证分析有助于理解各影响因素于绿色消费行为 的作用机制。不足之处在于, 引入研究的预设变量及 题量不足, 且定量研究方法无法帮助全面了解事物。 
因此, 后续研究中, 可面向消费者与酒店管理者, 通 过深度访谈深入理解各要素驱动机制。当前, 人类共 处于生态保护的十字路口, 践行绿色生产生活方式、 共建美好家园应成为全人类共同选择，因而围绕 “绿 色发展”、“绿色消费” 的相关研究也显得尤为必要, 且有待进一步深化。

\section{5. 结论}

(1) 探索性因子分析表明, 高星级酒店顾客绿色 消费行为影响因素含主观规范、感知利得、绿色消费 情境与感知利失。

(2) 与一般酒店公众相比, 高星级酒店顾客在一 般行为与积极行为都存在较高意愿。且主观规范对高 星级酒店顾客采纳绿色消费行为的影响特别显著。因 此, 营造特定的社会参照文化氛围对于促进高星级酒 店顾客绿色消费行为具有不容低估的作用。

(3) 感知利得、感知利失分别呈现显著正向与显 著负向影响, 且集中体现了高星级酒店顾客绿色消费 行为的内部心理归因。人们关注实施某一特定行为对 自身利益的影响, 一方面, 配合与支持绿色消费可能 存在环境改善、符合群体规范、节省费用等好处; 另 一方面, 实施绿色消费行为可能会带来体验感下降 (如带来麻烦、舒适度下降等), 这有悖于高星级酒店 顾客的基本消费诉求。因此, 提升个体对酒店绿色消 费措施的配合与支持应建立在精细化的传播沟通与 及时的服务承诺上, 并且后续措施的布施可以借助 “登门槛技术” 理念, 在消费者接受了实行障碍较小 或成本较低的行为后, 再实行障碍较大或成本较高的 行为。

（4）一般行为与积极行为的塑造路径中, 绿色消 费情境也需考虑。绿色消费情境对 “配合酒店绿色政 策推行”与 “入住认证的绿色酒店” 有显著正向影 响。因此, 利用酒店空间, 增强绿色消费知识与绿色 消费产品的展示、传播与教育, 可促使个体意识到绿 色消费措施推行的目标和意义, 进而采取支持行为。

\section{REFERENCES}

[1] UNWTO. (2021) Hotel Energy Solutions. https://www.unwto.org/hotel-energy-solution.

[2] Chen K, Gao G. (2019) Study on the Meaning of Green Living and its Improvement Mechanism. Studies on Socialism with Chinese Characteristics, 06:92-98.

[3] Xie T. (2016) Consumers' Intention to Visit Green Hotels: Based on TPB Perspective. Tourism Tribune. 31(06):94-103.

[4] Choi H, Jang J, Kandampully J. (2015) Application of the extended VBN theory to understand consumers' decisions about green hotels. International Journal of Hospitality Management.
51:87-95.

[5] Verma V K , Chandra B . (2017) An application of theory of planned behavior to predict young Indian consumers' green hotel visit intention. Journal of Cleaner Production. 172(PT.1):1152-1162.

[6] Han H . (2015) Travelers' pro-environmental behavior in a green lodging context: Converging value-belief-norm theory and the theory of planned behavior. Tourism Management. 47(apr.):164-177.

[7] Kang K H , Stein L, Heo C Y, et al. (2012) Consumers' willingness to pay for green initiatives of the hotel industry. International Journal of Hospitality Management. 31(2).

[8] Mas'od, Adaviah, Chin T A .(2014) Determining Socio-demographic, Psychographic and Religiosity of Green Hotel Consumer in Malaysia. Procedia Social and Behavioral Sciences. 130:479-489.

[9] Zhao L M, Zhang H B, Sun J H. (2015) Factors influencing the public low-carbon consumption behavior in hotels - Based on the survey of Tianjin hotel customers. Journal of Arid Land resources and Environment. 29(4):53-58.

[10] Li Z P. (2009) A Study on Customers' Green Consumptive Behaviors in Tourist Hotels. Tourism Tribune. ,24(08):34-39.

[11] Nimri R, Patiar A, Kensbock S . (2017) A green step forward: Eliciting consumers' purchasing decisions regarding green hotel accommodation in Australia. Journal of Hospitality and Tourism Management. $33: 43-50$.

[12] Hair J F, Black W C, Babin B J, et al. (2010) Multivariate Data Analysis: A Global Perspective, 7th ed. Pearson Prentice Hall. The New Jersey

[13] Wu M L. (2010) Statistical Analysis of Questionnaires: SPSS Operation and Application. Chongqing University Press, Chongqing.

[14] Staats H J, Wit A P, Midden C. (1996) Communicating the Greenhouse Effect to the Public: Evaluation of a Mass Media Campaign from a Social Dilemma Perspective. Journal of Environmental Management. 46(2): 189-203.

[15] Tajfel H., Turner J.C. (1986) The Social Identity Theory of Intergroup Behavior. Political Psychology. 13 (3): $276-293$

[16] Wang J M, He A Z. (2011) Psychological Attribution and Policy Paths of Consumer's Low Carbon Consumption Behavior: An Exploratory Research Based On Grounded Theory. Nankai Business Review, 14(04):80-89+99. 